\title{
Assessment of Radiobiological $\alpha / \beta$ Ratio in Lung Cancer and Fibroblast Cell Lines Using Viability Assays
}

\author{
ILIAS V. KARAGOUNIS, ELENI K. SKOURTI, MARIA V. LIOUSIA and MICHAEL I. KOUKOURAKIS
}

Department of Radiotherapy/Oncology, Democritus University of Thrace, Alexandroupolis, Greece

\begin{abstract}
Background/Aim: Altered fractionation is an area of intense clinical research in radiation oncology. Estimation of the $\alpha / \beta$ ratio of individual carcinomas after establishment of primary cell cultures from tumor biopsies may prove of importance in the individualization of radiotherapy schemes. Materials and Methods: Here we proposed a simple method to estimate the $\alpha / \beta$ ratio in cultured cell lines (two lung carcinomas: $A 549$ and H1299; one lung fibroblast cell line: MRC5), using viability assays. Results: For the A549 cell line, the $\alpha / \beta$ ratio ranged from 14-25 Gy, for H1299 from 11-43 Gy and for the MRC5 fibroblast cell line this was far lower, ranging from 0.69 to $6 \mathrm{~Gy}$. The $\alpha / \beta$ ratio decreased when extracted from comparisons of lower dose per fraction schemes. Conclusion: The $\alpha / \beta$ ratio of a cell line can be easily defined after simple viability/dose fractionation experiments.
\end{abstract}

The molecular biology of radiation-induced cell death remains poorly elucidated. Apoptosis and mitotic catastrophe are the two identified patterns of death after irradiation (1). According to the linear-quadratic model, radiation death results as the contribution of two different components (2). The first one is the direct creation of non-reparable lethal DNA damage (the $\alpha /$ linear component) and the second is the accumulation of non-lethal events ( $\beta /$ exponential component) that become lethal when more than one are combined. Which of the two components is more important strongly depends not only upon the cell and tissue type, but also upon the radiation dose and the quality of the beam. An $\alpha / \beta$ ratio characterizes each cell type, and reflects the dose in Grays where both components contribute equally to the overall cell lethality.

This article is freely accessible online.

Correspondence to: Michael I. Koukourakis, MD, Department of Radiotherapy - Oncology, Democritus University of Thrace, Alexandroupolis 68100, Greece. Tel: +30 2551074622, Fax: +30 2551030349, e-mail: targ@her.forthnet.gr

Key Words: Lung cancer cell lines, lung fibroblasts, $\alpha / \beta$ ratio.
Low $\alpha / \beta$ values characterize normal cells and tissues (such as fibroblasts and endothelial cells) which are less sensitive to small radiation fractions, while the damage suffered increases rapidly with the increase of the dose per fraction. In contrast, high $\alpha / \beta$ values are typical of highly proliferating normal cells (such as mucosa and skin epithelium) and the damage induced depends strongly on the total physical dose applied and weakly on the fractionation of the radiation dose (3). Cancer cells and tissues have long been considered to behave like highly proliferating normal cells, therefore being credited with high $\alpha / \beta$ ratios. This assumption, however, has proven to be incorrect, as radiobiological analysis of clinical data in prostate and breast cancer have shown low $\alpha / \beta$ values for these common tumors $(4,5)$. Assays that could provide the $\alpha / \beta$ values of carcinomas in patients before irradiation would allow the individualization of the fractionation scheme, providing schedules that would be the most appropriate for each patient.

In the current study, we sought to characterize the $\alpha / \beta$ ratio in two human lung cancer cell lines and a fibroblast cell line using simple dose-response experiments and radiobiological analysis of the biological isoeffect dose. This would provide a model for subsequent application in primary cell lines established from patients with cancer.

\section{Materials and Methods}

Cell lines/culture. A549 (human lung adenocarcinoma), H1299 (human non-small cell lung carcinoma), and MRC5 (fibroblast cell line) all from Cell Line Service, GmbH (Eppelheim, Germany) were used for the experimental procedures. Cells were cultured under aseptic conditions using Dulbecco's modified Eagle's medium as basal medium) (Gibco, Waltham, MA USA) supplemented with $10 \%$ fetal bovine serum (Biosera), 100 units $/ \mathrm{ml}$ penicillin and 100 $\mu \mathrm{g} / \mathrm{ml}$ streptomycin (Gibco, Nuaille, France), and $2 \mathrm{mM} \mathrm{L-}$ glutamine (Gibco). Cells were maintained at standard conditions $\left(37^{\circ} \mathrm{C}, 5 \% \mathrm{CO}_{2}\right)$ in humidified atmosphere and were used upon reaching $70-80 \%$ confluency.

Cell irradiation and dose/viability curves. The methodology applied to deliver a range of radiation doses to a single 96-well plate has 
been described and validated elsewhere (8). A PRECISE (ELEKTA, Stockholm, SE) 6-MV linear accelerator with multileaf collimator was used in this study The 6-MV photon energy produced has a maximum dose depth of $16 \mathrm{~mm}$ water and a tissue phantom ratio $\left(\mathrm{TPR}_{20,10}\right)$ of 0.680 .

A549, H1299 (250 cells/well) and MRC5 (500 cells/well) cells were seeded in a 96-well plate and irradiated with $0,2,4,6,9$ and $12 \mathrm{~Gy}$. Cell viability was assessed utilizing AlamarBlue ${ }^{\circledR}$ at 6 and 8 days post irradiation (before surviving cells start their rapid regrowth (6) and before control cells have increased to the upper detectable limit beyond which the AlamarBlue ${ }^{\circledR}$ method cannot provide cell growth estimates). Thereafter, the dose/viability curves were created and the isoeffect dose $D_{0}$ value (dose that allows $37 \%$ viability at 8 th day post irradiation) was estimated.

Fractionation schemes. Three fractionation schemes were applied: i) one fraction delivering $\mathrm{D}_{\mathrm{o}} \mathrm{Gy}$; ii) two fractions delivering $\mathrm{D}_{\mathrm{o}} / 2$ Gy $24 \mathrm{~h}$ apart; and iii) three fractions delivering $\mathrm{D}_{\mathrm{o}} / 3 \mathrm{~Gy} 12 \mathrm{~h}$ apart. Considering that quasi full repair of the reparable damage is accomplished within $8 \mathrm{~h}$, these two fractionation schemes allowed full recovery between fractions. The single fraction scheme was applied at $0 \mathrm{~h}$. In this way, all fractionation schemes delivered the dose within the same overall treatment time.

AlamarBlue $^{\circledR}$ assay. The AlamarBlue ${ }^{\circledR}$ assay (ThermoScientific, Waltham, MA USA) is a reliable method for measuring cell viability (6). This assay, using the metabolic activity of cells to reduce resazurin (oxidized form: 7-hydroxy-3H-phenoxazin-3-1-10-oxide) to resorufin, counts the number of cells with active mitochondria, as resazurin reduction is achieved by mitochondrial enzymes (7). The fluorescence of these two forms is measured at $530-560 \mathrm{~nm}$ as excitation wavelength and at $590 \mathrm{~nm}$ emission wavelength. Thus, this assay constitutes a simple, reliable and safe method while it is nontoxic to the cells

Determination of cell death. For the evaluation of the AlamarBlue ${ }^{\circledR}$ assay, cells were seeded in a 96 -well plate at a density of 250 (for A549 and H1299) or 500 (for MRC5) cells/well. AlamarBlue ${ }^{\circledR}(10 \% \mathrm{v} / \mathrm{v})$ was added to each well. As negative control (background measurement), we used culture medium without cells. Vitamin $\mathrm{C}(0.2 \mathrm{mg} / \mathrm{ml})$ was used for full reduction of resazurin $(5 \mu \mathrm{l} /$ well $)$ so that all measurements were adjusted to the fluorescence of the fully reduced resazurin. The relative fluorescence (in RFU units: well minus background units) was measured every $30 \mathrm{~min}$ for $12 \mathrm{~h}$, using a microplate reader (FLUOstar ${ }^{\circledR}$ Omega; BMG LABTECH GmbH, Ortenberg, Germany). Six values for each time point were obtained and the mean was used to plot the dose-response curves (6).

Cell growth/viability of irradiated and control (non-irradiated) cells was calculated and plotted every 2 days, using the AlamarBlue assay. In this way, viability-time curves were created for each cell line. The viability ratio for a given time point $\left(\mathrm{V}_{\mathrm{t}}\right)$ and fractionation scheme was calculated using the relative fluorescence units (RFU) provided by the AlamarBlue assay, as follows:

$\mathrm{V}_{\mathrm{t}}=\left(\mathrm{RFU}_{\text {irradiated cells }}-\mathrm{RFU}_{\text {negative control }}\right) /$

$\left(\mathrm{RFU}_{\text {non-irradiated cells }}-\mathrm{RFU}_{\text {negative control }}\right)$

The death induced by a fractionation scheme at a given time point (Dt) was then calculated using the formula: $\mathrm{D}_{\mathrm{t}}=100-\mathrm{V}_{\mathrm{t}}$
Biological effective dose (BED) and $\alpha / \beta$ ratio calculation. The BED for each fractionation scheme was calculated according to the following formula: $\mathrm{BED}=D(1+\mathrm{dx} /(\alpha / \beta))$, where $D$ is the total physical dose, $\mathrm{d}$ is the applied fraction and, $\alpha / \beta$ is the $\alpha / \beta$ ratio we wish to estimate (9). If for a given cell line fractionation scheme A leads to a\% growth reduction compared to the non-irradiated cells on a specific post-irradiation day, and scheme B leads to $\mathrm{b} \%$ effect, then the ratio of the BEDs will be equal to the ratio of the growthreduction percentages. Thus,

$\mathrm{BED}_{\mathrm{A}} / \mathrm{BED}_{\mathrm{B}}=\left(\alpha / \beta+d_{\mathrm{A}}\right) /\left(\alpha / \beta+d_{\mathrm{B}}\right)$,

i.e. $\%$ growth reduction by scheme $\mathrm{A} / \%$ growth reduction by scheme $\mathrm{B}$, where growth reduction refers to comparison against the growth rate of non-irradiated cells. From this equation, the $\alpha / \beta$ ratio can be calculated.

Graph design. Graph presentation and curve plotting was performed usingGraphPad Prism 5.00 version (GraphPad Software Inc., San Diego, CA, USA)

\section{Results}

The dose/viability curves of the three cell lines are shown in Figure 1. Using these curves, the $\mathrm{D}_{\mathrm{o}}$ value (dose that allows $37 \%$ viability at 8 th day post irradiation) was estimated. Therefore, the isoeffect dose was 5.2 Gy, 6.7 Gy and 2.9 Gy for the A549, H1299 and the MRC5 cell lines, respectively. By splitting these Do values into two (2.6, 3.35 and $1.45 \mathrm{~Gy}$, respectively for the three cell lines) and into three fractions (1.73, 2.23 and $0.97 \mathrm{~Gy}$, respectively), we derived three fractionation schemes of an equal physical dose for each cell line. Subsequently, cells were irradiated with these three fractionation schemes, as described in the Materials and Methods.

Cell growth/viability assessment of irradiated and control (non-irradiated) cells started immediately daily. In this way, viability-time curves were created for each cell line, showing the growth rates in control cells and for the three fractionation schemes applied (Figure 2).

Using the percentage death from Figure 2, noted on day 6 and on day 8 , we calculated $\alpha / \beta$ ratios for the three cell lines (Table I). For the A549 lung cancer cell line, the $\alpha / \beta$ ratio ranged from 14-25 Gy, for the H1299 from 11-43 Gy and for the MRC5 fibroblast cell line this was far lower, ranging from 0.69 to $6 \mathrm{~Gy}$. Calculation based on the 3-fraction vs. 1fraction schemes provided lower $\alpha / \beta$-values for all three cell lines examined compared to calculations based on 2-fraction vs. 1-fraction schemes.

\section{Discussion}

In the current study, we sought to establish a simple method for calculating the $\alpha / \beta$ ratio of cell lines based on cell viability following irradiation with three different fractionation schemes (1 vs. 2 vs. 3 fractions). From the viability-time curves, it is 
a

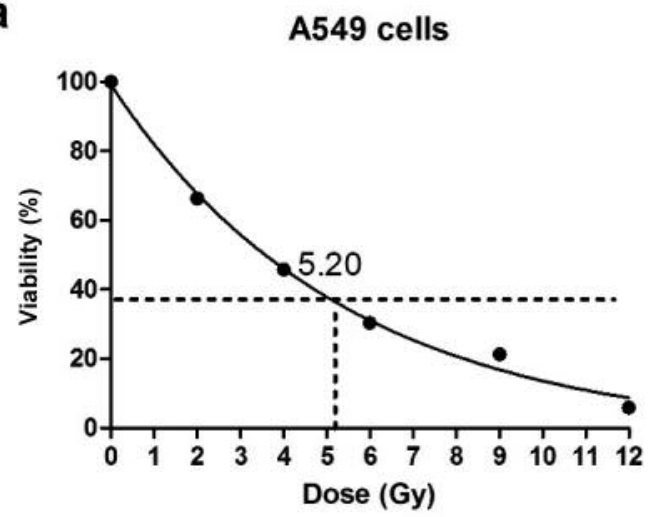

b
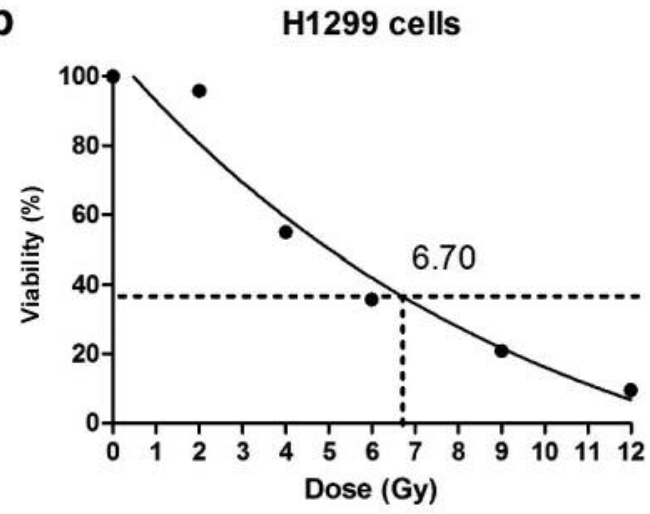

C

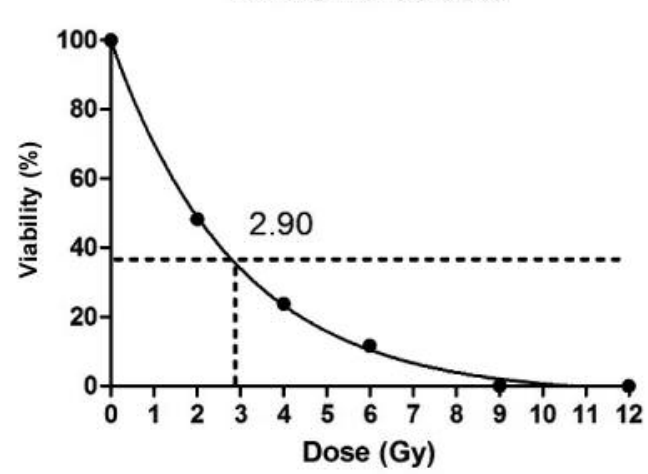

Figure 1. Dose-viability response curves for the three cell lines studied. Dotted lines indicate the calculation of the $D_{o}$ value in $G y$.

evident that cell viability increased with increasing the number of fractions. This was expected, as repair of reparable damage occurs within the interfraction intervals. The viability of cells was better in the 3 -fraction scheme, showing that the repair is more intense when smaller fractions are applied. Presumably, by increasing the dose per fraction, a larger amount of nonreparable damage occurs, leading to reduced viability.
Table I. The $\alpha / \beta$ ratio values obtained for the three cell lines, according to the fractionation schemes and to the of assessment of cell viability.

\begin{tabular}{lccc}
\hline Cell line & $\begin{array}{c}\text { Fractionation } \\
(\text { Gy/fraction } \times \text { no. of fractions })\end{array}$ & Day 6 & Day 8 \\
\hline A549 & $1.73 \times 3$ vs. $5.2 \times 1$ & 14 & 21 \\
& $2.6 \times 2$ vs. $5.2 \times 1$ & 18 & 25 \\
H1299 & $2.23 \times 3$ vs. $6.7 \times 1$ & 11 & 17 \\
& $3.35 \times 2$ vs. $6.7 \times 1$ & 28 & 43 \\
MRC5 & $0.97 \times 2$ vs. $2.9 \times 1$ & 0.69 & 1.33 \\
& $1.45 \times 3$ vs. $2.9 \times 1$ & 2.34 & 6 \\
\hline
\end{tabular}

Comparing the $\mathrm{D}_{0}$ values among the three cell lines examined, the H1299 cancer cell line was more radioresistant compared to A549. Lung fibroblasts were far more sensitive than the cancer cells. However, the $\alpha / \beta$ ratio for fibroblasts (0.6-6 Gy) was far lower compared to that for the cancer cells (11-43 Gy), compatible with the overall clinical and experimental estimation of the $\alpha / \beta$ values in connective tissues (fibrosis), which is around 4 Gy $(5,9-11)$. A recent study suggested an $\alpha / \beta$ value of $1.3 \mathrm{~Gy}$ for lung fibrosis, as extracted from clinical data (12). Moreover, clinical data suggest that lung cancer $\alpha / \beta$ values ranging from 10 to $20 \mathrm{~Gy}$ are more appropriate for the prediction of dose response in hypofractionated radiotherapy, which is in accordance with the high $\alpha / \beta$ values we found herein with viability experiments in lung cancer cell lines (13). As the $\alpha / \beta$ values of the cancer cell lines were several fold higher compared to fibroblasts, it is concluded that fibroblast sensitivity increases disproportionally with increasing the dose per fraction as compared to cancer cells lines.

An interesting point in the current study was that the $\alpha / \beta$ ratio decreased when extracted from comparisons of lower dose per fraction scheme with the one-hit fraction. This again may be a result of increasing reparability of cells irradiated with low doses. The ability to repair radiation damage seems not to be linear, but rather follows an exponentially increased effectiveness with decreasing dose per fraction. In this way, the larger the difference between the fractions applied in two schemes, the smaller the $\alpha / \beta$ ratio becomes, increasing the damaging impact of large fractions compared to the smaller fractions. This finding demands further evaluation as it may be of value in clinical practice, where standard $\alpha / \beta$ values are considered for normal tissues to estimate the equivalence between hypofractionation schemes and standard fractionation. The biological equivalence of very large fractions may be underestimated if the $\alpha / \beta$ value for normal fibroblasts decreases on widening the dose difference (e.g. 2 vs. 4 Gy per fraction compared to $2 v s$. 8 Gy per fraction). Very large fractions may demand far lower $\alpha / \beta$ ratios for a correct estimation of the equivalence between fractionation schemes. 
a

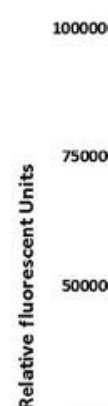

00000
A549

750000

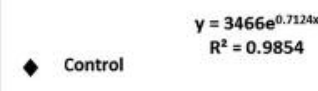

- $1 \times 5.2 \mathrm{~Gy} \quad \mathrm{y}=3306.9 \mathrm{e}^{0.44385 x}$

A $2 \times 2.6 \mathrm{~Gy}$

$x \quad 3 \times 1.76 y$

$R^{2}=0.9932$
$y=3328.9 e^{0.5085 x}$
$\mathrm{R}^{2}=0.9939$

$y=3565.5 e^{0.5245 x}$

$\begin{aligned} & \\ R^{2} & =0.9927\end{aligned}$

250000

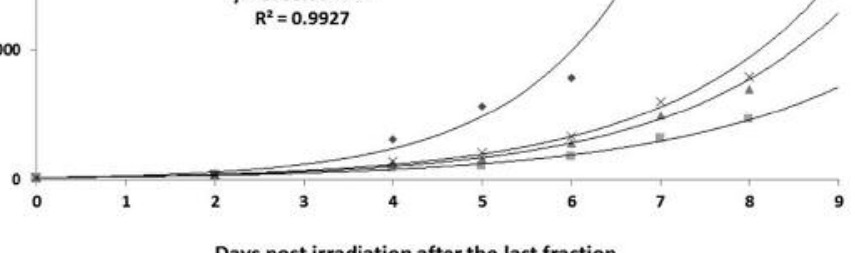

Days post irradiation after the last fraction

b

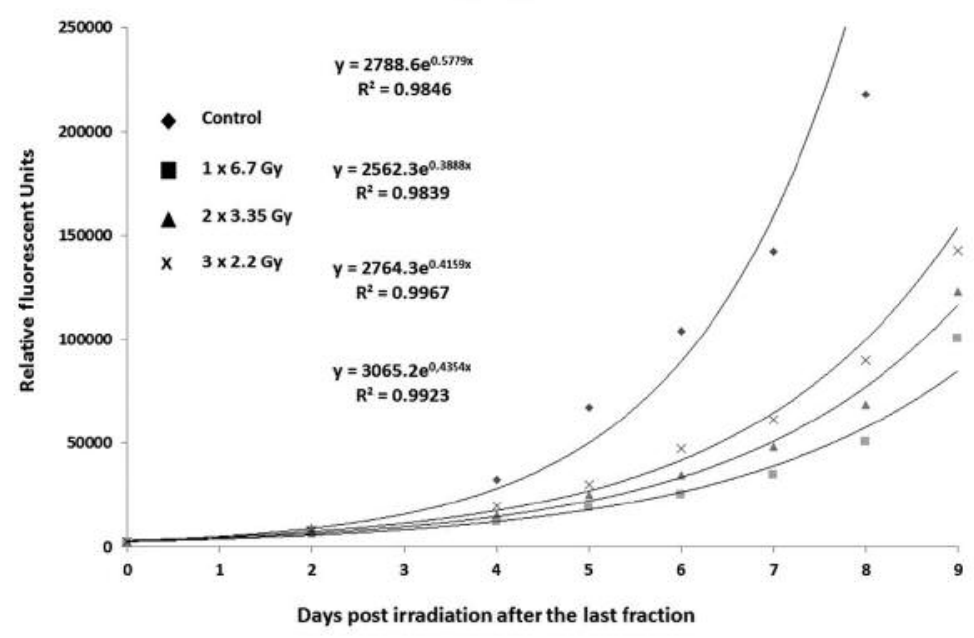

C

MRC5

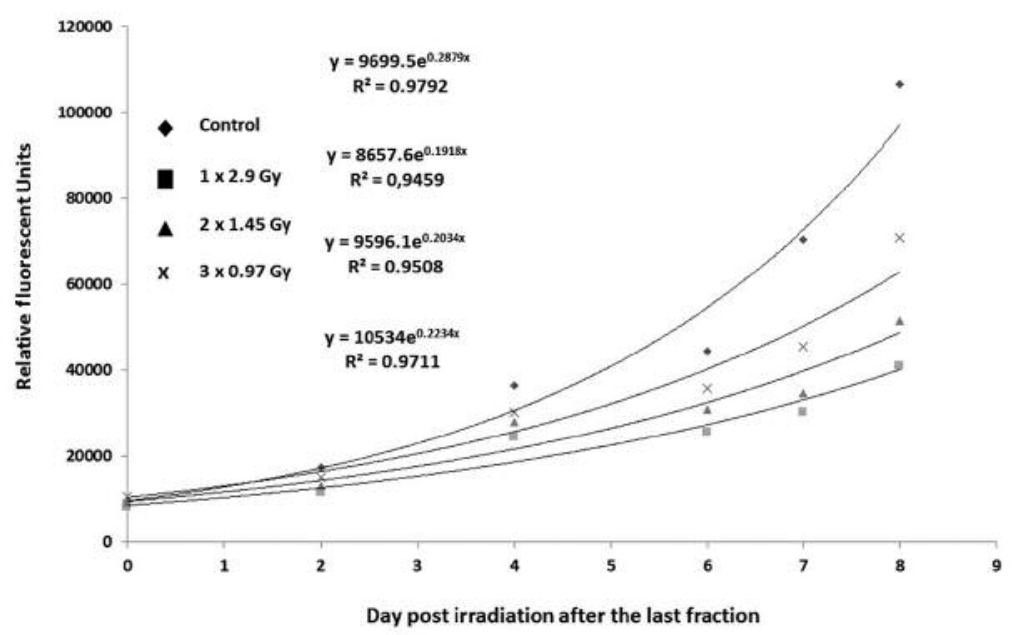

Figure 2. Non-linear regression analysis of growth-time curves for the three cell lines studied, following irradiation with $D_{o}$ Gy. Each line corresponds to the growth rate noted without radiation and after the three fractionation schemes applied. 
We conclude that the $\alpha / \beta$ ratio of a cell line can easily be defined after simple viability-dose fractionation experiments. A pilot study to establish primary cancer cell cultures from patients undergoing biopsy of the tumor and, subsequently, to calculate the $\alpha / \beta$ ratio before radiotherapy is ongoing.

\section{Acknowledgements}

The study was financially supported by the Tumour and Angiogenesis Research Group.

\section{References}

1 Eriksson D and Stigbrand T: Radiation-induced cell death mechanisms. Tumour Biol 31: 363-372, 2010.

2 Cadwick KH and Leenhouts HP: The Molecular Theory of Radiation Biology. Monograph on Theoretical and Applied Genetics 5. Springer-Verlag, New York, 1981

3 Thames HD and Hendry HR: Fractionation in radiotherapy. Taylor and Francis, London, 1987.

4 Fowler J, Chappell R and Ritter M: Is $\alpha / \beta$ for prostate tumors really low? Int J Radiat Oncol Biol Phys 50: 1021-1131, 2001.

5 START Trialists' Group, Bentzen SM, Agrawal RK, Aird EG, Barrett JM, Barrett-Lee PJ, Bliss JM, Brown J, Dewar JA, Dobbs HJ, Haviland JS, Hoskin PJ, Hopwood P, Lawton PA, Magee BJ, Mills J, Morgan DA, Owen JR, Simmons S, Sumo G, Sydenham MA, Venables K and Yarnold JR: The UK Standardisation of Breast Radiotherapy (START) Trial A of radiotherapy hypofractionation for treatment of early breast cancer: a randomised trial. Lancet Oncol 9: 331-341, 2008.

6 Zachari MA, Chondrou, PS, Pouliliou SE, Mitrakas AG, Abatzoglou I, Zois CE and Koukourakis MI: Evaluation of the AlamaBlue assay for adherent cells irradiation experiments. Dose Response 12: 246-258, 2013.
7 Page B, Page M and Noel C: A new fluorometric assay for cytotoxicity measurements in vitro. Int J Oncol 3: 473-476, 1993.

8 Abatzoglou I, Zois CE, Pouliliou S and Koukourakis MI: Establishment and validation of a method for multi-dose irradiation of cells in 96-well microplates. Biochem Biophys Res Commun 431: 456-459, 2013.

9 Jones B, Dale RG, Deehan C, Hopkins KI and Morgan DA: The role of biologically effective dose (BED) in clinical oncology. Clin Oncol (R Coll Radiol) 13: 71-81, 2001.

10 van Rongen E, Madhuizen HT, Tan CH, Durham SK and Gijbels MJ: Early and late effects of fractionated irradiation and the kinetics of repair in rat lung. Radiother Oncol 17: 323-337, 1990.

11 Bentzen SM and Overgaard M: Relationship between early and late normal-tissue injury after postmastectomy radiotherapy. Radiother Oncol 20: 159-165, 1991.

12 Scheenstra AE, Rossi MM, Belderbos JS, Damen EM, Lebesque JV and Sonke JJ: Alpha/beta ratio for normal lung tissue as estimated from lung cancer patients treated with stereotactic body and conventionally fractionated radiation therapy. Int $\mathrm{J}$ Radiat Oncol Biol Phys 88: 224-228, 2014.

13 Chi A, Wen S, Liao Z, Xu J, Nguyen NP, Welsh JS and Komaki $\mathrm{R}$ : What would be the most appropriate $\alpha / \beta$ ratio in the setting of stereotactic body adiation therapy for early-stage non-small cell lung cancer? Biomed Res Int 2013: 391021, 2013. 